\title{
A critical analysis on deeply bound kaonic states in nuclei
}

\author{
E. Oset ${ }^{a, b}$ and H. Toki ${ }^{b}$ \\ ${ }^{a}$ Departamento de Física Teórica and IFIC, Centro Mixto Universidad de Valencia-CSIC, \\ Institutos de Investigación de Paterna, Aptd. 22085, 46071 Valencia, Spain \\ ${ }^{b}$ Research Center for Nuclear Physics, Osaka University, Ibaraki, Osaka 567-0047, Japan
}

November 10, 2018

\begin{abstract}
We make a critical analysis on the theoretical calculations that lead to predictions of deeply bound kaonic states in nuclei. The model set-up, after dropping several important processes and channels, leads unavoidably to an unrealistic deep potential with a very small imaginary part. We review also the experimental results taken as reference for the claim of deeply bound kaons. We suggest that the peaks of the proton spectra come from $K^{-}$absorption on a pair of nucleons, leaving the rest of the nucleons as spectators. Based on this conjecture we predict what would happen in other nuclei.
\end{abstract}

\section{Introduction}

The search for deeply bound hadronic states has a long history. Deeply bound pionic atoms were predicted theoretically by using various optical potentials and the widths were expected to be smaller than the separation between the neighboring levels, which should make a clear experimental case [1, 2, 3]. The unexpected small widths are due to the presence of a large repulsive s-wave pion-nucleus interaction, which pushes away pions from the nuclear interior to avoid pion absorption. After several trials, the pionic states were found in the $\left(d,{ }^{3} \mathrm{He}\right)$ reaction [4, 5] on the basis of the suggestion given in Ref. [6, 7] and far less clearly in the $\left(\pi^{-}, \gamma\right)$ reaction [8] suggested in Ref. [9]. The history for the possible $\eta$ bound states is also long [10, 11, 12, 13, 14, 15]. Although most optical potentials lead to bound states, they also share the unpleasant feature that the widths are wider than the separation of the levels, which makes the experimental identification difficult. In spite of this difficult experimental condition, claims have been made that a $\eta$ bound state in ${ }^{3} \mathrm{He}$ can be identified [16], although the association of the experimental signal to a bound $\eta$ state is not completely unambiguous [17. Hence, it is important for the observation 
of deeply bound hadronic states the existence of a mechanism to make the widths of the deeply bound hadronic states smaller than the separation between the neighboring levels.

The other hadron that has attracted attention recently is the kaon. Kaonic atoms have been phenomenologically studied, and the large strength of the potential assumed at a time raised hopes that deeply bound states could exist [18. However, the imaginary part of the optical potential is large and therefore the widths of the deeply bound kaonic states come out to be too large to be detected as distinguished states. Hence, one should rely on the microscopic derivation of the kaon optical potential to find a mechanism for small widths of deeply bound kaonic states, if they exist. By deeply bound states we mean states bound by about $40-200 \mathrm{MeV}$. Note that with realistic potentials at small densities one obtains in heavy nuclei states bound by a few $\mathrm{MeV}$, which have not been observed and which are narrow enough to be distinguishable from other levels with the same angular momentum [19, 20, 21, 22].

The microscopic derivation of the optical potential for kaonic atoms is related strongly to the properties of the $\Lambda(1405)$ state, which is located just below the kaon-proton threshold. There have been many studies on the theoretical derivation of kaon nucleus optical potential. We shall discuss the details of the recent studies on the $\Lambda(1405)$ state and the density dependence of the kaon-nucleon interaction in the nuclear medium later on in Sect. 2 .

The discussion on the kaons in nuclei became more interesting due to the prediction made by Akaishi and Yamazaki on the existence of extremely deep kaonic states 24. They consider the $\Lambda(1405)$ state as the bound state of the kaon and proton and its width is caused by the coupling to the pion- $\Sigma(1193)$ channel. Assuming for the interactions a gaussian form with a fixed width, they obtained the interaction parameters by fitting the mass of the $\Lambda(1405)$ and the kaon-nucleon interaction at threshold. The kaon-nucleon interaction in the isospin $\mathrm{I}=0$ channel comes out to be strongly attractive and the imaginary part becomes zero below the pion- $\Sigma$ threshold. This model set-up leads to the existence of very deep and quite stable kaonic states in ${ }^{3} \mathrm{He}$ and ${ }^{4} \mathrm{He}$ systems. At the same time, the large attractive interaction between the kaon and the nucleons makes the system very compact as much as ten times the nuclear density. The width becomes then small, since the deeply bound kaonic states come out to be below the pion- $\Sigma$ threshold. The width comes solely from a weak coupling of the deeply bound kaonic states to the pion $\Lambda$ channel with the isospin $\mathrm{I}=1$.

This proposal of the existence of deeply bound kaonic states, which have large nuclear density, triggered experimentalists to detect the kaonic states in light nuclei. Suzuki et al. made experiments by using stopped $\mathrm{K}^{-}$on ${ }^{4} \mathrm{He}$ and measured protons in coincidence with pions [25]. They identified some peak structure in the proton spectra. If the peak structure is identified to be caused by the formation of a strange tribaryon, the mass is $3115 \mathrm{MeV}$ and the width is about $20 \mathrm{MeV}$. In recent talks [26] it has been claimed that the strange tribaryon should be identified with a deeply bound kaonic state although the isospin of the state is different from the original prediction, since other possibilities were concluded unlikely to provide the peak structure in the proton spectrum. The formation probability of such an exotic strange tribaryon state is surprisingly large, of order of one 
percent, for stopped negative kaons, even though the theoretical model of Ref. 24] requires high density objects for deeply bound kaonic states, which would lead naturally to small transition nuclear matrix elements.

In this paper, we would like to make a critical analysis of the theoretical approach, which leads to the prediction of deeply bound kaonic states in light nuclei and at the same time review the recent experimental results. For this purpose, we shall present the theoretical development of the kaon-nucleon interaction in free space in the framework of the chiral unitary model and the modification of the interaction due to the many body effects in the nuclear medium in Sect. 2. In the free space (kaon-nucleon system), the coupled channel effect of various channels leads to a more complex structure for the $\Lambda(1405)$ state. In the nuclear medium, the two body kaon absorption process becomes significant with density. We shall then discuss the simplified treatment of the kaon-nucleon interaction and its modification in the nuclear medium done in Ref. 24] in view of the recent development of the chiral unitary model in Sect. 3. In Sect. 4, we review the experimental results from various different points of view for the interpretation of the claimed deeply bound kaonic state. Sect. 5 will be devoted to the summary.

\section{Kaon-nucleon interaction and the kaon-nucleus op- tical potential}

It is very important to understand the kaon-nucleon interaction and its modification due to the nuclear medium, which leads to the kaon-nucleus optical potential, for the discussion of deeply bound kaonic states. In particular, the understanding of the $\Lambda(1405)$ is essential in order to study the fate of the kaons in the nucleus. The kaon-nucleon threshold is 1432 $\mathrm{MeV}$, which is $27 \mathrm{MeV}$ above the $\Lambda(1405)$ state. Hence, the $\Lambda(1405)$ should get a strong influence from the kaon-proton interaction. The width of the $\Lambda(1405)$ is about $50 \mathrm{MeV}$, decaying exclusively into the pion- $\Sigma(1193)$ system. Hence, the minimum ingredients for the structure of the $\Lambda(1405)$ are the kaon-nucleon interaction and its coupling to the pion- $\Sigma$ channel. Another feature, which should be taken care for the development of the kaonnucleon dynamics, is that the kaon-nucleon scattering amplitude is repulsive at threshold. This feature is well known both from extrapolation of scattering data [27], and from the measurement of kaonic atoms in the proton [28].

By taking a suitable dynamical model, we can make the amplitude for the $K^{-} p$ channel to have a typical resonance shape. The peak of the imaginary part appears about $20 \mathrm{MeV}$ below the $K^{-} p$ threshold, and the real part changes sign at the same energy, changing from an attractive interaction below the resonance peak to repulsion above the peak. As a consequence of it (counting also the $K^{-} n$ interaction) the low density $K^{-}$optical potential, $t \rho$, is repulsive at the $K^{-} p$ threshold, where $t$ is the kaon-nucleon T-matrix and $\rho$ is the nuclear density. The scattering T-matrix is obtained by solving the Bethe Salpeter equation and hence the iteration of diagrams shown in Fig. 1 is summed up.

It is interesting to discuss what happens when the $K^{-}$interacts with nucleons in a nu- 


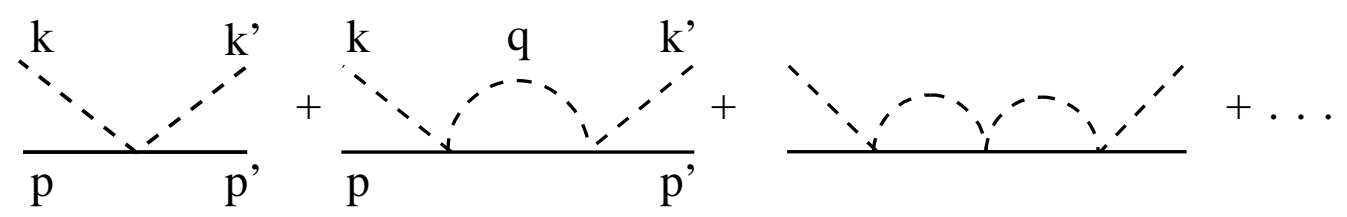

Figure 1: Diagrammatic representation of the Bethe Salpeter equations in $\bar{K} N$ scattering, where the solid line denotes the nucleon and the dashed one the kaon.

clear medium. In the intermediate states (loops in the scattering series of Fig. 1), there are $\bar{K} N$ states among other channels. In the presence of the medium the nucleon intermediate states have to be placed above the Fermi sea due to the Pauli blocking effect. This implies that the formation of the $\Lambda(1405)$ resonance requires more energy, as a consequence of which the resonance shape is reproduced now at higher energies. Hence, as one can see in Fig.2, when the real part of $t$ is moved at higher energies, the zero of the resonant scattering amplitude would cross the $K^{-} p$ threshold and now the interaction is attractive at the $K^{-} p$ threshold. This intuitive picture describes what happens in the calculations [29] and is the main reason to convert the repulsive interaction of the low density limit into an attractive one as soon as a density which produces a sensible Pauli blocking builds up. A potential depth of around $200 \mathrm{MeV}$ for the kaons can be obtained in this way [24.

We should not stop here, however. The kaon feels now a strong attractive potential and the kaon also appears in the intermediate states of the Bethe Salpeter series. With the strong attraction now on the kaon, its excitation requires less energy, and hence using the same arguments as before, the resonance is produced at a smaller energy. Once again the zero of the real part of the scattering amplitude moves to lower energy below the $K^{-} p$ threshold, leading once more to repulsion. The presence of the resonance is thus of extreme importance to determine the fate of the interaction in the medium and, in view of the shift up and down of the resonance when medium corrections are taken into account, a reliable calculation requires selfconsistency in the kaon selfenergy, in the sense that one must determine the kaon selfenergy, replace it in the kaon propagators, reevaluate the kaon selfenergy, replace it again in the kaon propagator and so on till convergence is achieved.

This selfconsistency procedure was done for the first time in Ref. [30]. It was found that the attraction felt by the kaons was drastically reduced. Subsequent calculations [31, 32], including those which consider also the renormalization of the intermediate pions in the pion- $\Sigma$ channel 33] were performed and the strength of the potential was shown to be attractive and of the order of $40-50 \mathrm{MeV}$ at nuclear matter density. This selfconsistency procedure, including also the p-wave excitation of $\Lambda h$ and $\Sigma h$ components by the kaon, or $p h$ excitation by the pion, automatically produces the kaon absorption by two nucleons, $K^{-} N N \rightarrow N \Lambda, N \Sigma$. There is hence a reduction of about a factor four for the real part of the kaon optical potential and there appears the imaginary potential due to two body absorption process, when the selfconsistency is implemented. No calculation which neglects these important effects caused by the selfconsistency procedure should be deemed realistic.

All the features discussed above are worked out more systematically in the $\mathrm{SU}(3)_{f}$ chiral unitary model. The $\Lambda(1405)$ resonance is dynamically generated in the coupled channel 


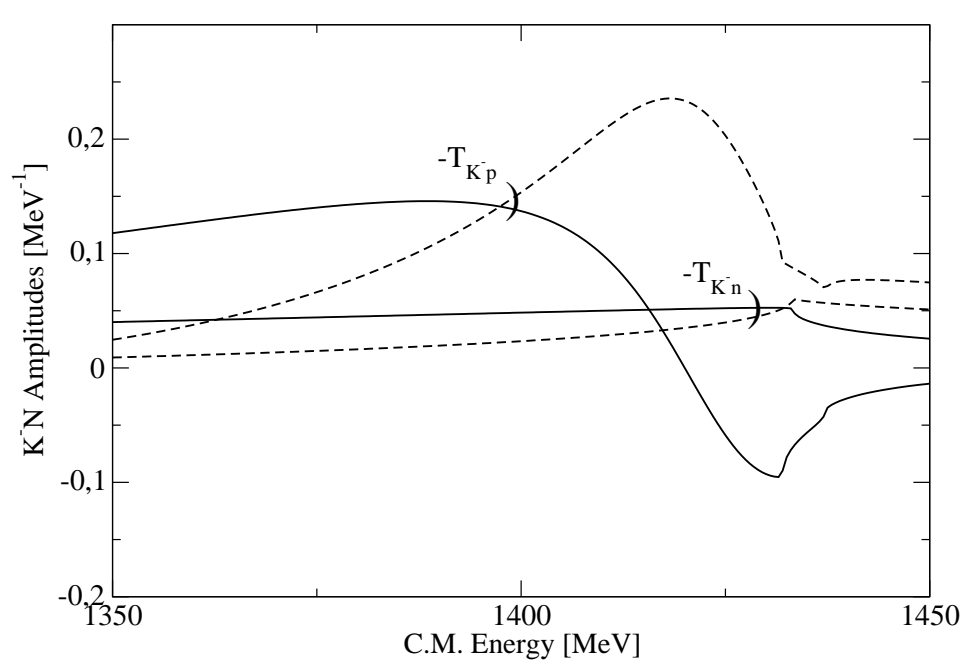

Figure 2: Scattering amplitudes for $K^{-} p \rightarrow K^{-} p$ and $K^{-} n \rightarrow K^{-} n$ around and below the $K^{-} p$ threshold in the chiral unitary model. The solid curves are the real part and the dashed ones the imaginary part for $\mathrm{K}^{-} \mathrm{p}$ and $\mathrm{K}^{-} \mathrm{n}$ channels [36].

model of $\bar{K} N, \pi \Sigma$ [34] and in the chiral unitary model [35, 36, 37, 38, 39], where one uses input from chiral Lagrangians and, in addition to the $\bar{K} N, \pi \Sigma$ channels, one also has other channels from the combination of the octet of pseudoscalar mesons with the octet of stable baryons. In these latter works the $\Lambda(1405)$ resonance is generated and a good reproduction of various $K^{-} p$ reactions is obtained.

The lowest order chiral lagrangian for the meson baryon interaction is given by

$$
L_{1}^{(B)}=<\bar{B} i \gamma^{\mu} \frac{1}{4 f^{2}}\left[\left(\Phi \partial_{\mu} \Phi-\partial_{\mu} \Phi \Phi\right) B-B\left(\Phi \partial_{\mu} \Phi-\partial_{\mu} \Phi \Phi\right)\right]>
$$

where $\Phi$ and $B$ are the ordinary SU(3) matrices of the meson and baryon fields respectively. From there, recalling the dominance of the $\gamma^{0}$ component at low energies, one deduces the kernel of the Bethe Salpeter equation (potential V)

$$
V_{i j}=-C_{i j} \frac{1}{4 f^{2}}\left(k^{0}+k^{\prime 0}\right)
$$

where $k^{0}, k^{\prime 0}$ are the energies of the mesons. The symmetric matrix $C_{i j}$, where $i, j$ stand for the indices of the coupled channels, $K^{-} p, \bar{K}^{0} n, \pi^{0} \Lambda, \pi^{0} \Sigma^{0}, \pi^{+} \Sigma^{-}, \pi^{-} \Sigma^{+}, \eta \Lambda, \eta \Sigma^{0}, \bar{K}^{0} \Xi^{0}, K^{+} \Xi^{-}$ for charge zero, is given in Ref. [36]. The solution of the Bethe Salpeter equation, which sums up the diagrams of Fig. 1, is given by

$$
T=[1-V G]^{-1} V
$$

in the matrix form of the coupled channels.

We note here that one of the surprises of the chiral approach is that there are not just one $\Lambda(1405)$ but two states, and the shape obtained in experiments comes from a 


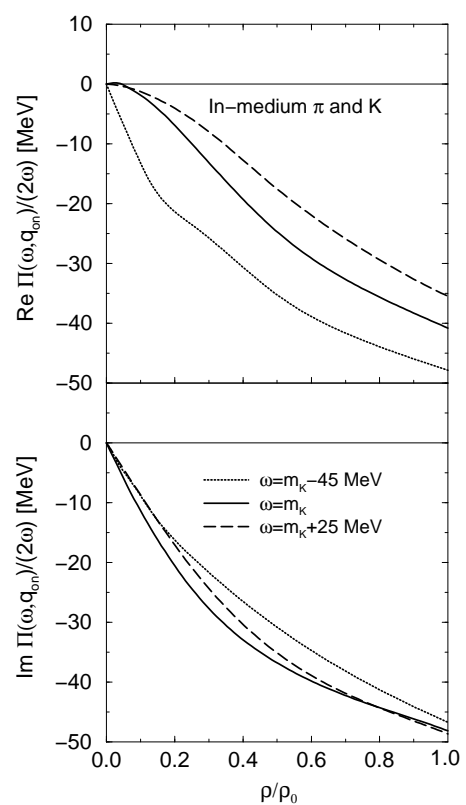

Figure 3: Real (top) and imaginary (bottom) parts of the $K^{-}$optical potential as a function of density obtained from the In-medium pions and kaons approximation. Results are shown for three different $K^{-}$energies: $\omega=m_{K}-45 \mathrm{MeV}$ (dotted curves), $\omega=m_{K}$ (solid curves) and $\omega=m_{K}+20 \mathrm{MeV}$ (dashed curves).

superposition of the two resonances with different weights for different reactions, which makes the position and width of the resonance vary from one reaction to another [40, 41. The existence of two resonances for $\mathrm{I}=0, \mathrm{~S}=-1$ was hinted in the work [37] and made clear in a more systematic manner in Ref. [42, where the existence of two octets and one singlet of dynamically generated resonances was established. Similar results were posteriorly found in Ref. [43]. The two $\Lambda(1405)$ states would correspond to the mixture of the singlet with one of the octets, the other octet combination moves up to generate the $\Lambda(1670)$, which was also reported before [44. These two $\Lambda(1405)$ states are quite different, there is one appearing around $1396 \mathrm{MeV}$, which has a width of about $140 \mathrm{MeV}$ and couples mostly to $\pi \Sigma$, while the other one appears around $1420 \mathrm{MeV}$, has a width about $30 \mathrm{MeV}$ and couples mostly to $\bar{K} N$. These features were also observed in two recent works which include the effect of higher order chiral Lagrangians [45, 46], although the width of the wide resonance is about $240 \mathrm{MeV}$ in Ref. [45].

With this elementary input and the corrections to the $\bar{K} N$ amplitude in the medium, the selfconsistent calculation of the $K^{-}$selfenergy in the nuclear medium done in Ref. 33. gives the results shown in Fig. 3 as a function of the nuclear density. The real part of the potential starts from a slightly positive value (repulsive) at zero density and switches its sign to negative (attractive) with increasing density. The strength of the attractive potential becomes larger as the $K^{-}$energy is increased. The imaginary part is essentially unchanged with the $K^{-}$energy. The direct use of this potential for the calculation of kaonic atoms of light and medium heavy nuclei provides a good reproduction of the kaonic 


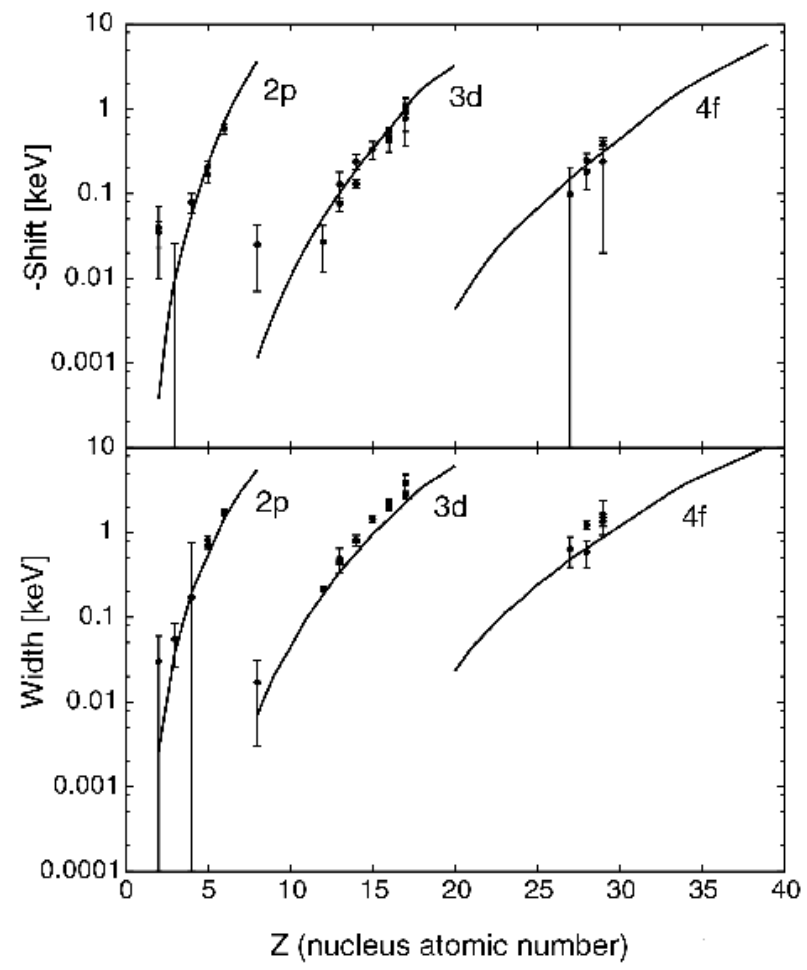

Figure 4: The energy shifts and the widths of the kaonic atoms in light and medium heavy nuclei [21. The experimental results are shown by the points with bars, which are compared with theoretical results obtained by using the optical potential obtained by the chiral unitary model shown in Fig. 3 [33].

atom data 21], as shown in Fig. 4. We can conclude here that the $\mathrm{SU}(3)$ chiral unitary model with the inclusion of all the medium corrections on the baryons and the mesons is supported from the kaonic atom data. Small diversions from the potential of [33] were found in 22] by performing a best fit to the kaonic data. The calculations of [32] also show similar values for the strength of the potential, and this is also the case of the $K^{-}$ optical potential evaluated in [23], based on a meson exchange model for the elementary $\bar{K} N$ interaction. In this latter work, higher partial waves, beyond the dominant s-wave intereraction were also considered wich had a moderate effect on the potential.

\section{Theoretical claim of deep potential}

In this section we discuss the work of Ref. 24], where claims of a deep $\bar{K} N$ potential with a small imaginary part are made which would provide very stable deeply bound kaonic states in nuclei. Quoting the authors textually, "we construct phenomenologically a quantitative $\bar{K} N$ interaction model that is as simple as possible using free $\bar{K} N$ scattering data, the KpX data of kaonic hydrogen and the binding energy and width of $\Lambda(1405)$, which can be 
regarded as an isospin $\mathrm{I}=0$ bound state of $\bar{K}+N$ ". They use as input $v_{\bar{K} N, \bar{K} N}, v_{\bar{K} N, \pi \Sigma}$, $v_{\bar{K} N, \pi \Lambda}$, which are fitted to data, and set $v_{\pi \Sigma, \pi \Sigma}, v_{\pi \Lambda, \pi \Lambda}$ equal zero "to simply reduce the number of parameters".

The assumptions made for the construction of the phenomenological $\bar{K} N$ interaction as stated above in Ref. 24 contain sufficient elements for a discussion here. As is well known from the coupled channel calculations leading to the $\Lambda(1405)$, (now we should go back to the two states) [35, 36, 37, 38, 39] the appearance of the two $\Lambda(1405)$ states 42 ] is a consequence of an intricate coupled channel dynamics, particularly the $\bar{K} N$ and $\pi \Sigma$, and none of the states can be claimed to be a bound state of $\bar{K} N$ as assumed in Ref. [24]. In Ref. 24] the rich coupled channel dynamics is destroyed from the moment that $v_{\pi \Sigma, \pi \Sigma}$ and $v_{\pi \Lambda, \pi \Lambda}$ are set equal zero. This is most dramatic when one realizes that the strength of the $\pi \Sigma \rightarrow \pi \Sigma$ in I=0 provided by the chiral Lagrangians is $4 / 3$ times bigger than that of $\bar{K} N \rightarrow \bar{K} N$ [36]. As a consequence, none of the resulting $\Lambda(1405)$ states qualifies as a bound $\bar{K} N$ state, as one can see by the large coupling of the two states to both the $\pi \Sigma$ and $\bar{K} N$ channels. If anything, even if still unacceptably rough, one should take the narrow state at $1420 \mathrm{MeV}$, which couples more strongly to $\bar{K} N$, as indicative of a bound $\bar{K} N$ state, in which case the strength of the interaction would be much smaller, since the state would be bound by only $12 \mathrm{MeV}$, in contrast with Ref. [24] where the binding energy of the $\bar{K} N$ is assumed to be $29.5 \mathrm{MeV}$. As a consequence of disregarding the coupled channel dynamics and assuming the $\Lambda(1405)$ as a $\bar{K} N$ bound state with such a large binding energy, the potential that is obtained from the fit is very large to begin with, the strength of the potentials at $r=0$ is of the order of $400 \mathrm{MeV}$ and the range of the interaction is also very small, of the order of $0.66 \mathrm{fm}$, which forces artificially the kaons to stick to the nucleons, producing nuclear densities with three baryons of the order of ten times the nuclear matter density at the center of the three baryon system in the calculation of Ref. 24].

This discussion indicates that the approximations done in Ref. [24] necessarily lead to larger scattering amplitudes than the chiral approaches. This is indeed the case as one can see by comparing Fig. 9 of Ref. [36] with Fig. 1 of Ref. 24]. To facilitate the comparison, the $\mathrm{I}=0$ amplitude plotted in Fig. 1 of Ref. [24] is equivalent to

$$
f^{I=0}=-\frac{1}{4 \pi} \frac{M}{\sqrt{s}}\left(2 t_{K^{-} p}-t_{K^{-} n}\right)
$$

with $t$ the amplitude used in Ref. [36].

We can see that in the plateau around $60 \mathrm{MeV}$ below the $\bar{K} N$ threshold the chiral amplitude is about $2.4 \mathrm{fm}$ in Ref. [36], while that of Ref. [24] is about $5 \mathrm{fm}$. One may argue that the $\bar{K} N$ amplitude below the threshold is not an observable and one would like to have a closer experimental evidence of the failure of the model of Ref. [24]. Such an observable can be seen in the recent experiment of the reaction $K^{-} p \rightarrow \pi^{0} \pi^{0} \Sigma$ in the region of excitation of the $\Lambda(1405)$ [41]. As one can see in the experiment the peak of the $\Lambda(1405)$ appears at $1420 \mathrm{MeV}$, while the one of Ref. 24] appears at $1403 \mathrm{MeV}$, the nominal value of the $\Lambda(1405)$ mass. The explanation given in Ref. 47] of the surprisingly large $\Lambda(1405)$ mass seen in Ref. [41] was natural within the context of the two $\Lambda(1405)$ states obtained in the chiral theories. Indeed, since the high energy $\Lambda(1405)$ state around 
$1420 \mathrm{MeV}$ couples mostly to $\bar{K} N$ the reaction $K^{-} p \rightarrow \pi^{0} \pi^{0} \Sigma$ gives more weight to this state in the amplitude, reflecting the most efficient mechanism to create the $\Lambda(1405)$ which is the emission of a $\pi^{0}$ prior to the scattering $K^{-} p \rightarrow \pi \Sigma$. Obviously, a theory with a unique $\Lambda(1405)$ at $1403 \mathrm{MeV}$ would not reproduce this experiment.

The $\bar{K} N$ interaction of Ref. 24] explained before is used to calculate the $g$ matrix in the nuclear medium, which is then used as a $K^{-}$nucleus optical potential. The Pauli blocking on intermediate states is implemented there and this converts the initial interaction into an attraction as we have explained above, only the strength obtained for kaon selfenergies around threshold is about 3.5 times bigger than the one obtained in Ref. [33, 32, 31]. The starting energy in the $g$ matrix calculation is changed to smaller values to account for the possibility of having kaons with smaller energy than free kaons (this is also done in Ref. [33], where the $K^{-}$selfenergy is calculated for several values of the starting $K^{-}$energy as shown in Fig. 3.). However, this binding energy is not used in the intermediate states in the scattering equations in Ref. 24] and the important effects of the selfconsistency explained above are simply lost, leading to an unrealistically large attractive potential.

In Ref. 24] this deep potential is used to explore the possibility of finding $K^{-}$bound states in light nuclei with $A=3$, and 4 . The same equation as in nuclear matter is used with an average Fermi momentum. It is easy to see from the equations used in section 3A of Ref. [24] that the Fermi momentum used there is equal or larger than that of nuclear matter at normal nuclear matter density. It is not intuitive that three or four nucleon systems have the same Pauli blocking effect than a full nuclear matter environment, but with this approximation and the former ones that lead to the unrealistically deep potential as we discussed, the potential for the $\mathrm{A}=3$ and 4 systems is evaluated which leads to binding energies of the kaon of the order of $70 \mathrm{MeV}$ with widths around $75 \mathrm{MeV}$, the imaginary part coming from $K^{-} N \rightarrow \pi \Sigma$.

At this point the authors argue that since the attraction due to the kaon is so large, it can induce a further contraction of the nucleus, increasing its density and consequently leading to more attraction on the kaon till the nuclear incompressibility stops the system from shrinking even further. With this mechanism, densities of the order of ten times the nuclear matter density in the center of the nucleus are obtained in Ref. [24]. This finding should be strongly questioned since to arrive to it they use the effective NN interaction 48, which accounts for the incompressibility of nuclear matter. Yet, at these high densities, and even much before, nuclear matter does not resemble this simple picture, and without entering into more complicated scenarios it is enough to quote that excitation of strange baryons would become favorable when matter is compressed with only three times or so of the nuclear matter density [49, 50, 51. In this direction it is also worth pointing out that, as matter compresses, the effect of three body forces becomes more relevant introducing a repulsion that leads to a stiffer equation of state ( a recent evaluation of such three body forces, that essentially produce a repulsive effect, can be seen in 52]). It has been pointed out that with a stiffer equation of state the $K^{-}$optical potential gets weekened [53, 54.

With these calculations the authors of Ref. [24] obtain deeply bound kaonic states bound by $108 \mathrm{MeV}$ in ${ }^{3} \mathrm{H}$ and $86 \mathrm{MeV}$ in ${ }^{4} \mathrm{H}$, with the widths of respectively 20 and 24 $\mathrm{MeV}$ due to the $\pi \Lambda$ decay channel since now the strong $\pi \Sigma$ decay channel is closed. This 
last issue opens a new discussion on the width of these states. In Ref. [24 the widths are considerably reduced by forcing the states to appear below the $\pi \Sigma$ threshold. Yet, in nuclear matter the two decay channels mentioned above are not the only ones and two body decay channels appear, like $K^{-} N N \rightarrow \Lambda N, \Sigma N, \Sigma(1385) N$. These two body decay channels and other multinucleon decay channels are automatically taken into account in the selfconsistent calculation of Ref. [33], but not in Ref. [24]. Instead of explicitly calculating the two body absorption processes, they are estimated in Ref. 24 by taking the empirical value of 16.5 percent branching ratio for the non pionic $K^{-}$absorption in ${ }^{4} \mathrm{He}$ [55. Then the authors apply the fraction of 17 percent to the imaginary part of the potential calculated in Ref. 32 which produces a contribution of $11 \mathrm{MeV}$ in the imaginary part of the potential for nuclear matter density, hence leading to a width of $22 \mathrm{MeV}$. However, the authors quote an approximate value of $12 \mathrm{MeV}$ for the two small nuclei. Here comes an observation to make which is that these two body mechanisms calculated in Ref. 33 are proportional to the nuclear density squared and hence, strictly speaking, at densities ten times the nuclear matter density as claimed in Ref. [24] would become 100 times larger, which means, inducing widths of $2000 \mathrm{MeV}$. Obviously this is a gross overestimate but invoking the "average" density of about three times nuclear matter density of Ref. 24] one should multiply this kaon absorption mechanism by a factor around ten, thus rendering the width of about $200 \mathrm{MeV}$, which is larger than the binding energy.

In the discussion mentioned above we have exploited the inconsistencies resulting from the chain of rough approximations done in Ref. 24] leads to. By contrast to that, the realistic calculations done in Ref. [31, 33] lead to deeply bound states in nuclei of the order of 10 and $30 \mathrm{MeV}$ in ${ }^{40} \mathrm{Ca}$ [21], but with the width of the order of $100 \mathrm{MeV}$. An independent work in Ref. [56] finds that in the event that bound states of kaons existed in medium nuclei in the region of $100-200 \mathrm{MeV}$, the widths would not be smaller than about $40 \mathrm{MeV}$ and these widths would be even larger in light systems with the claims of larger densities made in Ref. 24].

The developments of Ref. 24] have a continuation after the performance of an experiment at KEK 25]. This experiment claims to have found a strange tribaryon $\mathrm{S}(3115)$ which is seen as a peak in the spectra of emitted protons following the absorption of stopped $K^{-}$ in ${ }^{4} \mathrm{He}$. The authors of Ref. [25] do not mention in the paper the possibility that this state is the kaon bound system obtained in Ref. [24, since it would correspond to a $K^{-}$bound by $195 \mathrm{MeV}$ and it has necessarily $\mathrm{I}=1$, while the one predicted in Ref. [24 has a binding energy of $108 \mathrm{MeV}$ and is $\mathrm{I}=0$. Yet, in view of this experimental finding, the authors of Ref. 24] modify their approach by introducing relativistic effects, some contribution from spin-orbit interaction plus an ad hoc increase of the bare $\bar{K} N$ interaction by 15 percent, till a new $K^{-}$bound state by $195 \mathrm{MeV}$ appears [57. Let us quote, in connection to this, that in the chiral calculations of the optical potential of kaons in the medium of Ref. [30, 33, 31] the kaons were always treated relativistically and the study of the deeply bound $K^{-}$states in nuclei was done also relativistically using the Klein Gordon equation 21, as now used in Ref. [57. It is also worth quoting that after all the original rough approximations that induce further unreliable corrections from the nuclear shrinkage and the new relativistic corrections, the optical potential has acquired a strength of $618 \mathrm{MeV}$, with an imaginary 
part of $11 \mathrm{MeV}$. In contrast, the chiral calculations give a strength of $40-50 \mathrm{MeV}$, which would not give room for a further shrinkage of the nucleus since this is the strength of the nucleon nucleus potential, and the addition of a one more nucleon to a nucleus barely modifies its density. Furthermore, they have an imaginary part sizably larger, of the order of $50 \mathrm{MeV}$, due to the unavoidable many body decay channels.

\section{Discussion on the "experimental strange tribaryon state"}

On the basis of the discussion in the former section it is quite clear that the KEK experiment [25] could not be interpreted in terms of the creation of deeply bound kaonic states. From this perspective we would like to interpret the meaning of the peak seen there. The experiment is

$$
\text { stopped } \mathrm{K}^{-}+{ }^{4} \mathrm{He} \rightarrow \mathrm{S}+\mathrm{p}
$$

where $\mathrm{S}$ has a mass of about $3115 \mathrm{MeV}$, and has the quantum numbers of $Y N N$ with zero charge where $Y$ is a $\mathrm{S}=-1$ hyperon. The state $\mathrm{S}$ would have $I_{3}=-1$ and hence cannot be $I=0$ as predicted originally in Ref. [24], which was already noted in Ref. [25]. The peak is also quite narrow, around or smaller than $20 \mathrm{MeV}$. A tempting idea could be a bound state of $\pi \Lambda N N$, which, as noticed in Ref. 25, is only about $16 \mathrm{MeV}$ above the observed peak. Of course, after what has been learned in chiral unitary models [58, one would have to study this with coupled channels, which would make the identification as such a state possible only at a qualitative level. The width would come from pion absorption on two nucleons, which is expected large [59, 60].

There are, however, other potential explanations of the peak, which would deserve some attention. One of them was already pointed out in Ref. [25]. The peak of the proton comes at about $500 \mathrm{MeV} / \mathrm{c}$. There is a process that can create a peak at the same place. This is the reaction

$$
K^{-}+{ }^{4} \mathrm{He} \rightarrow{ }_{\Lambda}^{4} \mathrm{He}+\pi^{-}
$$

followed by

$$
{ }_{\Lambda}^{4} H e \rightarrow{ }^{3} H+p
$$

In this chain a pion with $255 \mathrm{MeV} / \mathrm{c}$ and a proton of $508 \mathrm{MeV} / \mathrm{c}$ would be emitted. This possibility is discarded in Ref. 25] in view of the following arguments:

1) The yield of this reaction is estimated to be of the order of $2 \times 10^{-4}$ according to Ref. 61], while the rate for the reaction of Eq. (5) is also estimated in Ref. 25] to be of the order of one percent. Actually, we do not find in Ref. 61] the estimate claimed in Ref. [25], and in Ref. 25] no reasons are given either for the yield of one percent claimed for the reaction of Eqs. (67), although, based on the experimental yields for hypernuclear formation 62 and the weight of the proton peak in Ref. 25 these assumptions look reasonable.

2) The authors of Ref. [25] make also a test to eliminate the possibility of the chain reaction by looking at the secondary pions. There a "fast $\pi$ " cut is made which would 
accept 90 percent of the $255 \mathrm{MeV} / \mathrm{c}$ pions. If the proton peak came from the chain reaction it would appear in connection with the "fast $\pi$ " cut. However, the peak is seen both in the "fast $\pi$ " cut case as well as in the complementary set of data with about the same intensity, which would exclude the interpretation of the peak as coming from the chain reaction. There could be a caveat in the former argument since the experiment is using a thick target of superfluid helium of $15 \mathrm{~cm}$ long and $23.5 \mathrm{~cm}$ of diameter at a density of 0.145 $\mathrm{g} / \mathrm{cm}^{3}$. Indeed, the pions of $255 \mathrm{MeV} / \mathrm{c}$ are on top of the $\Delta$ resonance region and hence the cross sections are large. In fact, by looking at the experimental cross sections of the paper of Ref. [59] one induces a quasielastic cross section for $\pi^{-}{ }^{4} \mathrm{He}$ in the region of interest of about $190 \mathrm{mb}$. It is easy then to estimate that about five percent of the pions would undergo quasielastic collisions and due to Pauli blocking they would lose energy because they need to transfer momentum to the nucleons to excite them on top of the Fermi sea. The result of this exercise, however, is that not enough pions would lose energy to make 50 percent of the signal appear outside the "fast $\pi$ " cut. Hence, based on the tests made in Ref. 25] and the former discussion, we do accept their conclusion that the peaks are not due to the chain reaction of Eqs. (67). Nevertheless, it would be very interesting to have the pion spectrum in coincidence with the proton peak in order to learn more about the reaction.

The former reflexions gain a new dimension when one realizes that a similar proton peak is seen in the experiment at FINUDA in different nuclei from ${ }^{6} \mathrm{Li}$ at around 510 $\mathrm{MeV} / \mathrm{c}$ 64. While the interpretation of these states as deeply bound kaonic states would lead to the unlikely result that the $K^{-}$binding energy is about the same for different nuclei from three to five to more baryons, the alternative explanation as creating strange multibaryon system with the same binding energy for all nuclei, would not be less surprising. Such systematics in different nuclei deserve a serious thought to see a clear explanation. Certainly, a chain reaction with the formation of a $\Lambda$ hypernucleus and a posterior decay through nonmesonic decay into a proton and a bound normal nucleus could, a priori, be a likely explanation for the peaks, but the tests done in Ref. [25] give not much room for hope in this direction. Nevertheless, it would still be interesting to measure the spectrum of the pions in coincidence, for the reasons given above.

\section{Suggested mechanism}

In view of the previous unsuccessful trials let us explore the reaction which is most likely to happen: This is $K^{-}$absorption by two nucleons in ${ }^{4}$ He leaving the other two nucleons as spectators. This kaon absorption process should happen from some $K^{-}$atomic orbits which overlap with the tail of the nuclear density and hence the Fermi motion of the nucleons is small. Then we would have $K^{-} N N \rightarrow \Lambda N$, and $\Sigma N$, and the two baryons are emitted back to back with the momentum for the proton of 562 , and $488 \mathrm{MeV} / \mathrm{c}$ respectively. These results are very interesting: the peak of the proton momentum in Ref. [25, before proton energy loss corrections, appears at $475 \mathrm{MeV} / \mathrm{c}$ (see Fig. 5 of this reference). This matches well with the $488 \mathrm{MeV} / \mathrm{c}$ proton momentum from a $K^{-} N N \rightarrow \Sigma N$ event, and the proton 
would lose about $13 \mathrm{MeV} / \mathrm{c}$ when crossing the thick target. This energy loss is compatible with the estimate of about $30 \mathrm{MeV} / \mathrm{c}$ in Ref. [25], particularly taking the width of the peak also into account.

This suggestion sounds good, but then one could ask oneself: what about $K^{-} N N \rightarrow$ $\Lambda N$ ? Should not there be another peak around $550 \mathrm{MeV} / \mathrm{c}$, counting also the energy loss? The logical answer is yes, and curiously one sees a second peak around $545 \mathrm{MeV} / \mathrm{c}$ in the experiment. The peak is clearly visible although less pronounced than the one at 475 $\mathrm{MeV} / \mathrm{c}$ and it appears in the region of fast decline of the cross section.

There are other arguments supporting our suggestion. Indeed, as mentioned above, the pion momenta from $\Lambda$ decay are smaller than those from $\Sigma$ decay. As a consequence of this we should expect the peak associated with $p \Lambda$ emission to appear in the low momentum side of the pion (the range of pion momenta is from $61 \mathrm{MeV} / \mathrm{c}$ to $146 \mathrm{MeV} / \mathrm{c}$ from phase space considerations). Actually, this is the case in the experiment of Ref. 25] as one can see in Fig. 5d of this reference, corresponding to the spectrum when the low pion cut is applied, where the peak of higher momentum stands out more clearly. On the other hand, by working out the phase space for $\Sigma$ decay, the pion momenta range from $162 \mathrm{Mev} / \mathrm{c}$ to $217 \mathrm{MeV} / \mathrm{c}$ and the pion could be seen in the two regions of pion momenta of Ref. 25], as it is indeed the case (see figs. 5c, 5d of Ref. [25]).

One can even argue about the size of the peaks and their relative strength. For this the information of Ref. [55] is very useful. There we find the following results for events per stopped $K^{-}$:

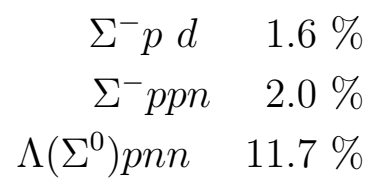

with errors of $30-40 \%$.

For the separation of the $\Lambda\left(\Sigma^{0}\right)$ contribution a rough approximation is done in Ref. [55], assuming that the $\Sigma^{0}$ yield is one half of the sum of that of the charged $\Sigma$ invoking isospin symmetry. In this case the 11.7 percent of Eq. (10) is split between 2.3 percent for $\Sigma^{0}$ and 9.4 percent for $\Lambda$. Isospin symmetry is not that simple with the system of four baryons in the final state and we estimate uncertainties by applying a different method. We can assume $K^{-} p p \rightarrow \Sigma^{0}(\Lambda) p$ proceeding via $K^{-} p \rightarrow \Sigma^{0}(\Lambda) \pi^{0}$ with the pion virtually exciting particle-hole states of the proton type. In this case we would have approximately the yields $\mathrm{Y}$ of the two processes as,

$$
\frac{Y\left(\Sigma^{0} N N\right)}{Y(\Lambda N N)}=\frac{\sigma\left(K^{-} p \rightarrow \pi^{0} \Sigma^{0}\right)}{\sigma\left(K^{-} p \rightarrow \pi^{0} \Lambda\right)}
$$

Next we use experimental information from Ref. 63] to estimate the ratio of Eq. (111). For a $K^{-}$of $100 \mathrm{MeV} / \mathrm{c}$ momentum the two cross sections are of the order of $20 \mathrm{mb}$. Using this information we count how many times we have $\Sigma p$ and $\Lambda p$ in the final state after $K^{-}$ absorption ( in the case of $\Sigma^{-} p d$ we naturally associate the $d$ with the spectator block) 
to find the probability to have a $Y p$ being the emitted pair, and we get about 3.7 percent for $\Sigma p$ and 3.1 percent for $\Lambda p$ when we use the partition of $\Sigma^{0}(\Lambda)$ from [55] and 4.88 percent versus 1.95 percent when we use Eq. (11), with experimental errors of the order of 50 percent or more. The absolute values for $\Sigma p$ emission make sense from the perspective that in Ref. 25. a rate of one percent for the production of the peak is estimated. A rate of one in four for the back to back emission of the pair and spectator remnant pair is realistic. On the other hand we see, that even with admitted large uncertainties, ratios of a factor 1.2 to 2.5 for $\Sigma p$ versus $\Lambda p$ come up from analysis of present data. A larger strength for the peak of lower proton momentum( $\Sigma p$ emission) relative to that of the smaller proton momentum ( $\Lambda p$ emission), of about a factor of two, is actually seen in Fig. $5(\mathrm{~d})$ of Ref. [25]. The $\Sigma^{-} p d$ final state of Eq. (8), with the spectator $d$ forming a bound state, should be one of the largest contributors to the $\Sigma^{-} p$ peak, leading to a momentum of the proton of $482 \mathrm{MeV} / \mathrm{c}$.

The hypothesis advanced should have other consequences. Indeed, this peak should not be exclusive of the small nuclei. This should happen for other nuclei. Actually in other nuclei, let us say $K^{-7} \mathrm{Li}$, the signals that we are searching for should appear when a proton as well as a $\Sigma$ or a $\Lambda$ would be emitted back to back and a residual nuclear system remains as a spectator and stays nearly in its ground state. We would thus expect two new features: first, the two peaks should be there. However, since now the spectator nuclear systems remain nearly in their ground states, only about the binding energy of the two participant nucleons will have to be taken from the kaon mass, instead of the $28 \mathrm{MeV}$ in ${ }^{4} \mathrm{He}$ for a full break up, as a consequence of which the proton momenta should be a little bigger. We make easy estimates of $502 \mathrm{MeV} / \mathrm{c}$ for the proton momentum in the case of $p \Sigma$ emission and $574 \mathrm{MeV} / \mathrm{c}$ for the case of $p \Lambda$ emission. Curiously the FINUDA data 64] exhibits two peaks in ${ }^{7} \mathrm{Li}$ around $505 \mathrm{MeV} / \mathrm{c}$ and $570 \mathrm{MeV} / \mathrm{c}$.

There is one more prediction we can make. The process discussed has to leave the remnant nucleus in nearly its ground state. This means that one has to ensure that the nucleus is not broken, or excited largely, when the energetic protons go out of the nuclear system. Theoretically one devises this in terms of a distortion factor that removes events when some collision of the particles with the nucleons takes place. Obviously this distortion factor would reduce the cross sections more for heavier nuclei and, hence, we should expect the signals to fade away gradually as the nuclear mass number increases. This is indeed a feature of the FINUDA data 64.

Similarly, we can also argue that the spectator nucleus, with a momentum equal to that of the combined pair on which $K^{-}$absorption occurs, will have smaller energies for heavier nuclei since their mass is larger. Hence, the spreading of the energy of the emitted proton should become narrower for heavier nuclei. This is indeed a feature of the FINUDA data 64 .

This sequence of predictions of our hypothesis, confirmed by the data of [25] and 64, provides a strong support for the mechanism suggested of $K^{-}$absorption by a pair of nucleons leaving the rest of the nucleons as spectators. Certainly, further tests to support this idea, or eventually refute it, should be welcome. An obvious test is to search for $\Sigma$ or $\Lambda$ in coincidence and correlated back to back with the protons of the peak. Awaiting further 
experimental information, we can say that after showing that the theoretical basis for the kaonic atoms hypothesis was a consequence of rough approximations which produced potentials one order of magnitude bigger than any realistic calculation, and after using information of the experiment of Ref. [25] which ruled out a likely two step process, by elimination we reached the present conclusion that, as we could prove, passes all tests of the experimental information provided by the KEK and FINUDA data.

\section{Conclusions}

In this paper we have made a thorough review of the theoretical developments that led to predictions of deeply bound kaonic atoms in light nuclei. We could show that there were many approximations done, which produced unreliably deep potentials. Two main reasons made the approximations fail dramatically: the problem of the coupled channels to produce the two $\Lambda(1405)$ states was reduced to only one channel, the $\bar{K} N$, and only one $\Lambda(1405)$, which was assumed to be a bound state of the $\bar{K} N$ potential was considered. The second serious problem was the lack of selfconsistency in the intermediate states, which makes the results absolutely unreliable when one is in the vicinity of a resonance, as is the case here. There were many other approximations but the former two are sufficient to obtain a potential as large as ten times what one gets without making these approximations. We also state that the width becomes zero in the $I=0$ channel due to the binding energy being lower than the pion- $\Sigma$ threshold. However, the selfconsistency consideration automatically produces the two body kaon absorption processes, which provides large widths and increases as the density squared.

With the weakness of the theoretical basis exposed and the realization that binding energies of $200 \mathrm{MeV}$ for a kaon in a system of three particles are out of scale, we looked for a plausible explanation of present experiments which could be interpreted differently than creating these deeply bound $K^{-}$states. After using information and the analysis of Ref. 25] discarding potential alternatives with chain reactions, we were led by elimination to a source of explanation deceivingly simple, and which, however, passes the present experimental tests: the association of the observed proton peaks to $K^{-}$absorption by a nucleon pair leaving the rest of the nucleons as spectators. From the emission of $p \Sigma$ we explained the peak found in Ref. 25] and from the emission of $p \Lambda$ we predicted another peak which is indeed present in the experiment of Ref. [25]. The hypothesis made led us to conclude that these peaks should also be visible in other nuclei at slightly larger proton momenta, should be narrower and their strength should diminish with increasing mass number of the nucleus. Fortunately, all these predictions could be tested with present data from FINUDA 64] and all the predictions were confirmed by these preliminary data.

Although further experiments could be advisable to further test our claims, it is also clear that the evidence provided here for these claims is by far larger than the one for deeply bound kaonic atoms, based exclusively on theoretical predictions that we have proved here to be largely overblown. Not to mention that the real predictions gave a binding energy half the one of the peak of Ref. [25], which would be assumed as a bound state of a 
kaon, and with the wrong isospin. The a posteriori corrections of the theory to match the experimental findings and increase the binding energy by a factor of two only added more uncertainties to the already unacceptably rough approach on which the genuine predictions were made.

\section{Acknowledgments}

We are grateful to Tadafumi Kishimoto and Takashi Nakano for fruitful discussions. We appreciate a careful reading of the present manuscript by Mamoru Fujiwara, Manuel Jose Vicente Vacas and Angels Ramos. This work is partly supported by DGICYT contract number BFM2003-00856, and the E.U. EURIDICE network contract no. HPRN-CT-200200311. This research is part of the EU Integrated Infrastructure Initiative Hadron Physics Project under contract number RII3-CT-2004-506078.

\section{References}

[1] H. Toki and T. Yamazaki, Phys. Lett. B 213 (1988) 129.

[2] H. Toki, S. Hirenzaki, T. Yamazaki and R. S. Hayano, Nucl. Phys. A 501 (1989) 653.

[3] J. Nieves, E. Oset and C. Garcia-Recio, Nucl. Phys. A 554 (1993) 509.

[4] T. Yamazaki et al., Z. Phys. A 355 (1996) 219.

[5] K. Itahashi et al., Phys. Rev. C 62 (2000) 025202.

[6] H. Toki, S. Hirenzaki and T. Yamazaki, Nucl. Phys. A 530 (1991) 679.

[7] S. Hirenzaki, H. Toki and T. Yamazaki, Phys. Rev. C 44 (1991) 2472.

[8] K. J. Raywood et al., Phys. Rev. C 55 (1997) 2492.

[9] J. Nieves and E. Oset, Phys. Lett. B 282 (1992) 24.

[10] R. S. Bhalerao and L. C. Liu, Phys. Rev. Lett. 54 (1985) 865.

[11] L. C. Liu and Q. Haider, Phys. Rev. C 34 (1986) 1845.

[12] H. C. Chiang, E. Oset and L. C. Liu, Phys. Rev. C 44 (1991) 738.

[13] S. A. Rakityansky, S. A. Sofianos, M. Braun, V. B. Belyaev and W. Sandhas, Phys. Rev. C 53 (1996) 2043.

[14] K. Tsushima, D. H. Lu, A. W. Thomas and K. Saito, Phys. Lett. B 443 (1998) 26 arXiv:nucl-th/9806043. 
[15] C. Garcia-Recio, J. Nieves, T. Inoue and E. Oset, Phys. Lett. B 550 (2002) 47 arXiv:nucl-th/0206024.

[16] M. Pfeiffer et al., Phys. Rev. Lett. 92 (2004) 252001 arXiv:nucl-ex/0312011.

[17] A. Sibirtsev, J. Haidenbauer, J. A. Niskanen and U. G. Meissner, Phys. Rev. C 70 (2004) 047001 arXiv:nucl-th/0407073.

[18] C. J. Batty, E. Friedman and A. Gal, Phys. Rept. 287 (1997) 385.

[19] E. Friedman and A. Gal, Phys. Lett. B 459 (1999) 43 arXiv:nucl-th/9902036.

[20] E. Friedman and A. Gal, Nucl. Phys. A 658 (1999) 345 arXiv:nucl-th/9907052.

[21] S. Hirenzaki, Y. Okumura, H. Toki, E. Oset and A. Ramos, Phys. Rev. C 61 (2000) 055205.

[22] A. Baca, C. Garcia-Recio and J. Nieves, Nucl. Phys. A 673 (2000) 335 arXiv:nucl-th/0001060.

[23] L. Tolos, A. Ramos, A. Polls and T. T. S. Kuo, Nucl. Phys. A 690 (2001) 547 arXiv:nucl-th/0007042.

[24] Y. Akaishi and T. Yamazaki, Phys. Rev. C 65 (2002) 044005.

[25] T. Suzuki et al., Phys. Lett. B 597 (2004) 263.

[26] M. Sato, talk at the Hadron Physics at COSY Workshop, Badhonnef (Germany), July 2005.

[27] A. D. Martin, Nucl. Phys. B 179 (1981) 33.

[28] M. Iwasaki et al., Phys. Rev. Lett. 78 (1997) 3067.

[29] V. Koch, Phys. Lett. B 337 (1994) 7 arXiv:nucl-th/9406030.

[30] M. Lutz, Phys. Lett. B 426 (1998) 12 arXiv:nucl-th/9709073.

[31] J. Schaffner-Bielich, V. Koch and M. Effenberger, Nucl. Phys. A 669 (2000) 153 arXiv:nucl-th/9907095.

[32] A. Cieply, E. Friedman, A. Gal and J. Mares, Nucl. Phys. A 696 (2001) 173 arXiv:nucl-th/0104087.

[33] A. Ramos and E. Oset, Nucl. Phys. A 671 (2000) 481 arXiv:nucl-th/9906016.

[34] R.H. Dalitz, T.C. Wong and G. Rajasekaran, Phys. Rev. 153 (1967) 1617

[35] N. Kaiser, T. Waas and W. Weise, Nucl. Phys. A 612 (1997) 297 arXiv:hep-ph/9607459. 
[36] E. Oset and A. Ramos, Nucl. Phys. A 635 (1998) 99 arXiv:nucl-th/9711022.

[37] J. A. Oller and U. G. Meissner, Phys. Lett. B 500 (2001) 263 arXiv:hep-ph/0011146.

[38] C. Garcia-Recio, J. Nieves, E. Ruiz Arriola and M. J. Vicente Vacas, Phys. Rev. D 67 (2003) 076009 arXiv:hep-ph/0210311.

[39] T. Hyodo, S. I. Nam, D. Jido and A. Hosaka, Phys. Rev. C 68 (2003) 018201 arXiv:nucl-th/0212026.

[40] D. W. Thomas, A. Engler, H. E. Fisk and R. W. Kraemer, Nucl. Phys. B 56 (1973) 15.

[41] S. Prakhov et al. [Crystall Ball Collaboration], Phys. Rev. C 70 (2004) 034605.

[42] D. Jido, J. A. Oller, E. Oset, A. Ramos and U. G. Meissner, Nucl. Phys. A 725 (2003) 181 arXiv:nucl-th/0303062.

[43] C. Garcia-Recio, M. F. M. Lutz and J. Nieves, Phys. Lett. B 582 (2004) 49 arXiv:nucl-th/0305100.

[44] E. Oset, A. Ramos and C. Bennhold, Phys. Lett. B 527 (2002) 99 [Erratum-ibid. B 530 (2002) 260] arXiv:nucl-th/0109006.

[45] B. Borasoy, R. Nissler and W. Weise, arXiv:hep-ph/0505239.

[46] J. A. Oller, J. Prades and M. Verbeni, arXiv:hep-ph/0508081.

[47] V. K. Magas, E. Oset and A. Ramos, Phys. Rev. Lett. 95 (2005) 052301 arXiv:hep-ph/0503043.

[48] A. Hasegawa and S. Nagata, Prog. Theor. Phys. 45 (1971) 1786

[49] J. Schaffner, C. B. Dover, A. Gal, C. Greiner and H. Stoecker, Phys. Rev. Lett. 71 (1993) 1328.

[50] I. Bombaci and B. Datta, Astrophys. J. 530 (2000) L69. arXiv:astro-ph/0001478.

[51] I. Vidana, I. Bombaci, A. Polls and A. Ramos, Astron. Astrophys. 399 (2003) 687. arXiv:astro-ph/0209068.

[52] M. M. Kaskulov, E. Oset and M. J. Vicente Vacas, Phys. Rev. C, in print. arXiv:nucl-th/0506031

[53] T. Maruyama, H. Fujii, T. Muto and T. Tatsumi, Phys. Lett. B 337 (1994) 19.

[54] J. Schaffner, A. Gal, I. N. Mishustin, H. Stoecker and W. Greiner, Phys. Lett. B 334 (1994) 268. 
[55] P. A. Katz, K. Bunnell, M. Derrick, T. Fields, L. G. Hyman and G. Keyes, Phys. Rev. D 1 (1970) 1267.

[56] J. Mares, E. Friedman and A. Gal, Phys. Lett. B $606 \quad$ (2005) 295 arXiv:nucl-th/0407063.

[57] Y. Akaishi, A. Dote and T. Yamazaki, Phys. Lett. B 613 (2005) 140 arXiv:nucl-th/0501040.

[58] J. A. Oller, E. Oset and A. Ramos, Prog. Part. Nucl. Phys. 45 (2000) 157 arXiv:hep-ph/0002193.

[59] D. Ashery, I. Navon, G. Azuelos, H. K. Walter, H. J. Pfeiffer and F. W. Schleputz, Phys. Rev. C 23 (1981) 2173.

[60] E. Oset, Y. Futami and H. Toki, Nucl. Phys. A 448 (1986) 597.

[61] H. Outa et al., Nucl. Phys. A 639 (1998) 251.

[62] H. Tamura et al., Phys. Rev. C 40 (1989) 479.

[63] J.K. Kim, Phys. Rev. Lett. 21 (1965) 29.

[64] N. Grion and S. Piano, private communication. See preliminary results in the talk of T. Yamazaki at the chiral05 Conference, Tokyo, 2005, http://chiral05.riken.jp/ 